\title{
INEQUALITIES FOR THE MINIMAL EIGENVALUE OF THE LAPLACIAN IN AN ANNULUS
}

\author{
A. G. RAMM AND P. N. SHIVAKUMAR
}

Abstract. We discuss the behavior of the minimal eigenvalue $\lambda$ of the Dirichlet Laplacian in the domain $D_{1} \backslash D_{2}:=D$ (an annulus) where $D_{1}$ is a circular disc and $D_{2} \subset D_{1}$ is a smaller circular disc. It is conjectured that the minimal eigenvalue $\lambda$ has a maximum value when $D_{2}$ is a concentric disc. If $h$ is a displacement of the center of the disc $D_{2}$ and $\lambda(h)$ is the corresponding minimal eigenvalue, then $\frac{d \lambda(h)}{d h}<0$ so that $\lambda(h)$ is minimal when $\partial D_{2}$ touches $\partial D_{1}$, where $\partial D$ is the boundary of $D$. Numerical results are given to back the conjecture. Upper and lower bounds are given for $\lambda(h)$.

Mathematics subject classification (1991): 35J05, 35P15.

Key words and phrases: Inequalities, estimation of eigenvalues, perturbation theory.

\section{REFERENCES}

[1] J. SoKolowsKi, J. ZolezIO, Introduction to shape optimization, Springer Verlag, Berlin, 1992.

[2] T. KATO, Perturbation theory for linear operators, Springer Verlag, Berlin, 1966. 